Teil III -

Datenschutz und Privatheit als Thema der Gesetzgebung und Medienregulierung 



\title{
Recht auf mein Selbst - Schutzräume kindlicher Entwicklungsphasen in der digitalen Gesellschaft
}

\author{
Stephan Dreyer
}

\section{Abstract}

Die digitale Gesellschaft datafiziert unweigerlich auch die Kindheit. Doch was bedeutet das für die gesetzliche Einhegung dieser besonderen Entwicklungsphase? Der Beitrag zeigt, dass das grundgesetzliche Recht auf freie Persönlichkeitsentwicklung kindbezogene Schutzräume (auch) im Digitalen umfasst. Dabei werden die Schutzgehalte eines spezifischen Kinderrechts auf Privatsphäre aus dem Recht auf freie Persönlichkeitsentwicklung und -entfaltung hergeleitet und dessen Besonderheiten und Ausprägungen in digitalen Medienumgebungen aufgezeigt. Durch den Abgleich verfassungsrechtlich erwünschter digitaler Schutzräume mit empirischen Einblicken in die datenschutzbezogenen Kenntnisse, Sorgen und Wünsche von Kindern und Jugendlichen zeigt der Beitrag bestehende Regelungslücken, aber auch die Herausforderungen bei ihrer gesetzlichen Ausfüllung auf. Denn die mögliche Umsetzung der Pflicht zur Schaffung gesetzlicher Schutzräume für Kinder im Digitalen weist strukturelle Herausforderungen und Spannungsfelder auf, etwa Datenschutz- oder Geheimnisinteressen der Minderjährigen, das Erziehungsrecht der Eltern sowie die begrenzte Steuerungsmacht sanktionsbewehrter Normen.

\section{Freie Persönlichkeitsentfaltung: Das Recht, sich selbst zu gehören}

Die Freiheit des Einzelnen wurde mit der Schaffung des Art. 2 Abs. 1 GG von einem vorgesetzlichen Naturrecht in eine positivrechtliche Form gegossen, die die Garantie der Menschenwürde aus Art. 1 Abs. 1 GG mit einer programmatischen Gewährleistung ergänzt: der prinzipiellen Freiheitsvermutung. Menschsein und Freiheit haben Vorrang vor staatlich verordnetem Dasein (Di Fabio 2019). Die Freiheit, die Art. 2 Abs. 1 GG garantiert, ist die freie Entfaltung der Persönlichkeit. Sie bezieht sich zum einen auf die Ebene der physischen Handlungsfreiheit, das heißt, jede Person darf prinzipiell tun und lassen, was sie will. Sie enthält aber auch eine psy- 
chische Komponente, die auf die innere Freiheit des Einzelnen abzielt: Kern dieser Freiheitsdimension ist das Allgemeine Persönlichkeitsrecht, das die Integrität der Persönlichkeit in geistig-seelischer Hinsicht und die soziale Identität schützt. Damit wird nicht eine bestimme Handlung oder ein bestimmtes Tun geschützt, sondern die Freiheit, nach eigenem Willen darüber zu entscheiden, was man tun oder nicht tun möchte. Dies rückt die jeder Handlung oder jedem Unterlassen vorausgehende innere Entscheidung des Einzelnen in den Fokus dieser Grundrechtsausprägung. Es geht um die Gewährleistung freier Selbstbestimmung in einem Zustand, in dem jede Entscheidungsfindung autonom und ohne fremde Einwirkungen erfolgen kann, in dem das Selbst, das eigene „Ich“ alleiniger Entscheidungshersteller und -träger ist. Damit umfasst das Recht auf freie Persönlichkeitsentfaltung zentral das Recht, sich selbst zu gehören. Der autonomiebezogene Schutz einer solchen entscheidungsbezogenen Lesart des Allgemeinen Persönlichkeitsrechts ist Vorbedingung für die Ausübung vieler weiteren Grundrechte, von der Wahrnehmung von Informations- und Kommunikationsgrundrechten über die Religionsfreiheit bis hin zur Versammlungsfreiheit.

Welche spezifischen Gehalte aber weist dieses Recht auf mein Selbst für Kinder und Jugendliche in einer digitalisierten, datafizierten Gesellschaft auf? Der Beitrag arbeitet die Notwendigkeit und Quelle eines spezifischen Persönlichkeitsentwicklungsrechts heraus (2.), zeigt anhand entwicklungspsychologischer Erkenntnisse und besonderer rechtlicher Umhegungen die derzeitigen Herleitungen und Ausgestaltungen besonderer Schutzräume von Kindern auf (3.) und setzt diese in Relation zu Formen informationeller Privatheit und Autonomie bei der digitalen Mediennutzung (4.). Schließlich wird aufgezeigt, dass für Kinder und Jugendliche aufgrund der begrenzten Kenntnisse der komplexen Datenverarbeitungspraktiken und personalisierten Selektionslogiken mit ihren vielfältigen Autonomiebezügen dringend auch die regulatorische Absicherung von Schutzräumen im Digitalen nötig ist, dass die Gesetzgebung sich hier aber teils komplexen Spannungsfelder gegenübersieht, die einfache Ansätze verbieten (5.).

\section{Unbeeinträchtigte Persönlichkeitsentwicklung als Vorbedingung freier Persönlichkeitsentfaltung}

Die Entfaltung des Selbst ist nur möglich, wenn sich zuvor ein Selbst entwickeln und Bahn brechen konnte. Das beschriebene Allgemeine Persönlichkeitsrecht geht implizit davon aus, dass es so etwas wie eine „Persönlichkeit" gibt, die sich durch Denken und Handeln nach innen und außen 
manifestiert. Dass jene Persönlichkeit aber nicht auf einmal dem Nichts entspringt, sondern das Ergebnis eines Prozesses ist, lässt sich aus Formulierungen wie einer „gelungenen Identitätsbildung“, der Idee einer „Entstehung des Selbst“ oder einer „Subjektwerdung“ (Becker 2017b) ablesen. Soll das Ergebnis eines solchen Prozesses die autonome Persönlichkeitsentfaltung sein, so muss sich jede Gewährleistung und Garantie der Freiheit der Entfaltung auch auf den vorherigen und andauernden Entwicklungsprozess beziehen: Die Vorbedingung der freien Entfaltung ist das Ergebnis einer freien Entwicklung.

Man wird nicht so weit gehen können, zu sagen, dass aus jeder fremdbestimmten oder manipulierten Entwicklungsphase zwingend eine unfreie Persönlichkeit entsteht - der Einzelne kann hier durchaus resilient sein, Fremdbestimmung bewusst als Eingriff werten und entsprechende Coping-Strategien entwickeln. Aus Sicht eines staatlichen Gewährleistungsauftrags kann aber jedenfalls nicht ausgeschlossen werden, dass eine Beeinträchtigung der freien Entwicklung sich so negativ auf die Ausbildung und Fortbildung von Persönlichkeitsfacetten auswirkt, dass der Prozess der Persönlichkeitsbildung nachhaltig gestört und - im schlimmsten Fall - zu einem Zustand führen kann, in dem eine freie Persönlichkeitsentfaltung gehemmt ist. Der staatliche Auftrag zur Gewährleistung der freien Persönlichkeitsentfaltung umfasst vor diesem Hintergrund auch den vorausgehenden Prozess der Persönlichkeitsentwicklung: Verfassungsrechtlich gesehen ist die freie Entwicklung der Persönlichkeit zwingende Voraussetzung für eine freie Persönlichkeitsentfaltung.

\section{Kindheit als besondere Phase der Persönlichkeitsentwicklung}

Dass sich das Grundgesetz in Art. 2 Abs. 1 GG auf die Persönlichkeitsentfaltung konzentriert, und damit eine irgendwie abgeschlossene Entwicklung impliziert, ist entwicklungspsychologisch längst überholt. Auch im (hohen) Erwachsenenalter formt und prägt sich unsere Persönlichkeit immer weiter aus; die (Weiter-)Entwicklung der eigenen Persönlichkeit ist ein lebenslanger Prozess. Dennoch unterscheidet sich die Kindheit substantiell von den späteren Phasen (Kap. 3.1). Das Recht umhegt diesen Zeitraum entsprechend in besonderer Weise (Kap. 3.2). 


\subsection{Kindheit als besondere biologische, psychologische und psychosoziale Entwicklungsphase}

In der Kindheit erscheinen viele Persönlichkeitsfacetten noch als dynamisch - das "Selbst“ ist hier in Teilen instabil, die eigene Persönlichkeit entsteht, wird in der sozialen Interaktion mit anderen ausprobiert und immer wieder angepasst. Teile der Persönlichkeit werden bewusst und unterbewusst verworfen, neue Facetten hinzugefügt. In der Kindheit und Jugend werden (Verhaltens-)Grenzen erkannt, aber auch ausgetestet, sozialadäquates Verhalten gelernt und die eigene Individualität, aber auch die von anderen beobachtet.

Die menschliche Entwicklung durchläuft dabei mehrere Stufen bzw. Konzepte des „Selbst“: Nach dem frühen Punkt des Selbst-Bewusstseins, in dem man den eigenen Körper als kontrollierbare biologische Einheit erkennt (Siegler et al. 2016: 410), entsteht nach und nach ein Selbst-Konzept. Das Selbst-Konzept erweitert die Eigenwahrnehmung um die kognitive Identitätskomponente, die über Generalisierungsprozesse aus situativen Selbstbewertungen entsteht und auf sprachliche Entwicklungen und Auseinandersetzungen angewiesen ist. Es entwickelt sich eine erste Gesamtheit der Einstellungen zur eigenen Person, deren Organisation sich durch immer wieder erfolgende Selbstbeurteilungen zunehmend ordnet. Später tritt dann ein Selbst-Wert(gefühl) dazu, bestehend aus der Generalisierung der affektierten Selbstbewertung und aus Kontrollüberzeugungen in Form einer handlungsbezogenen personalen Kontrolle. Diese Kontrolle ist Kern einer Identitätsentwicklung, bei der sich mit zunehmendem Alter und je nach konkreten Kontexten Verhaltensweisen ausdifferenzieren (Siegler et al. 2016: $412 \mathrm{ff}$.).

Die Phase des Heranwachsens zeichnet sich dabei durch die Instabilität des Selbst-Konzepts aus: Selbstbezogene Kognitionen und Evaluationen schwanken und verändern sich unter dem Einfluss einer Vielzahl sozialer und sozialpsychologischer Bedingungsgrößen. Bei älteren Jugendlichen stabilisieren sich diese Konzepte zunehmend (Greve/Thomsen 2019: 163), spätestens im Erwachsenenalter spricht die Entwicklungspsychologie von relativer Selbst-Konsistenz oder einer gefestigten Identität. Selbst-Konzepte weisen in unterschiedlichen sozialen Kontexten im frühen Kindheitsalter weniger, im Jugendalter umso mehr Varianzen in ihren generellen und spezifischen Aspekten auf: So sind Selbst-Konzepte bis weit in die Kindheit hinein stark positiv verzerrt (Siegler et al. 2016: 411). Erst später nimmt die Genauigkeit der Selbsteinschätzungen durch die allmähliche Integration auch negativer Informationen über eigene Fähigkeiten und Eigenschaften in das Selbstbild zu. Mit dieser Entwicklung einher geht die zunehmende 
Ausdifferenzierung des eigenen Rollenbildes; am Ende der Jugendzeit reflektiert das Selbstkonzept dann relativ stabile Überzeugungen und Werte.

Drei Dinge werden bereits anhand dieses sehr kurzen Überblicks deutlich: Selbst-Konzepte von Kindern und Jugendlichen sind hochgradig fluide, sie stabilisieren sich aber mit zunehmendem Alter. Dieser großen Veränderungen unterliegende Raum der Selbst-Entwicklung bildet sich bei Kindern und Jugendlichen aufgrund der Interaktion mit anderen weiter aus. Diese Interaktion ist Wirkfaktor im Sinne einer Sozialisations- und Entwicklungsinstanz: Persönlichkeitsentwicklung ist damit ein „permeabler Schutzraum auch des Sozialen“. Mit zunehmendem Kindesalter kann dabei das soziale Verhalten in unterschiedlichen sozialen und kommunikativen Kontexten unterschiedliche Ausprägungen aufweisen.

\subsection{Kindheit als rechtlich besonders umbegtes Lebensalter}

Die beschriebenen, fluiden Selbst-Konzepte bei Kindern und Heranwachsenden werden für einen besonderen gesetzlichen Schutz im Recht nicht ausdrücklich herangezogen. Sie zeigen sich aber in Form von Gesetzesbegründungen, die einem kindheitstheoretischen Konzept von Vulnerabilität anhängen (Andresen 2018). Als Gründe für den besonderen Schutz von Kindern und Jugendlichen finden sich regelmäßig deren geistige bzw. seelische Verwundbarkeit, ihre im Vergleich einfachere Beeinflussbarkeit, ihre Naivität oder Unwissenheit hinsichtlich komplexer wirtschaftlicher oder sozialer Prozesse, ihre begrenzte Fähigkeit zur Abschätzung von Handlungsfolgen oder ihre Impulsivität. Damit stellt die Gesetzgebung vordergründig vor allem auf begrenzte Erfahrungen und leichte Manipulierbarkeit durch äußere Reize oder Interaktionen statt. Auf den zweiten Blick aber geht es dem Recht darum, mögliche Enttäuschungen, Rückschläge, Verunsicherungen, Schäden und schlicht (rechtlichen oder elterlichen) Ärger, etwa beim Tappen in eine Abofalle, zu minimieren. Es geht um den Schutz vor Entscheidungen des Kindes, die sich negativ auf die Persönlichkeitsentwicklung auswirken können. Die oben dargestellte Herausbildung von Selbst-Konzepten steht so strukturell auch im Fokus des rechtlichen Kinderschutzes.

Mit Blick auf das Schutzgut einer freien, d.h. möglichst unbeeinträchtigten Selbst-Entwicklungsmöglichkeit und Selbst-Entfaltungsmöglichkeit nutzt das Recht dabei in unterschiedlichen Rechtsbereichen Altersgrenzen in Form rechtsfiktiver Entwicklungsstadien. Fiktionen sind im Recht ein oft genutztes Mittel, um einen ungewissen Sachverhalt rechtssicher zu regeln, indem gesetzlich eine bestimmte Tatsachenfolge verbindlich festge- 
schrieben wird. In Bezug auf Kinder und Jugendliche knüpfen rechtliche Fiktionen an bestimmte Altersgruppen und die dabei erreichten Entwicklungsstufen an, und sehen regelmäßig entsprechend abgestufte Rechtsfolgen vor. Im Folgenden einige Beispiele:

- Arbeitsschutz: Das Arbeitsrecht sieht kinderspezifische Normen vor, um die körperliche wie geistig-seelische Entwicklung von Kindern und Jugendlichen nicht durch schwere körperliche Arbeit oder Überforderung zu gefährden. Das Jugendarbeitsschutzgesetz (JArbSchG) geht vom Grundsatz eines Beschäftigungsverbots für Kinder und Jugendliche unter 15 Jahren aus (im Fall leichter Arbeit und mit Einwilligung der Erziehungsberechtigten: unter 14 Jahren). Ausnahmen für bestimmte Beschäftigungen von noch Jüngeren sind nur nach behördlicher Genehmigung möglich und unterliegen weitreichenden Anforderungen, darunter etwa ein aktuelles ärztliches Attest und Nachweise über Vorkehrungen zur Vermeidung einer Beeinträchtigung der körperlichen oder seelisch-geistigen Entwicklung. Auch in diesem genehmigungsbedürftigen Bereich sieht das Gesetz altersdifferenzierte Abstufungen vor. So dürfen Kinder zwischen drei und sechs Jahren maximal bis zu zwei Stunden täglich und nur in der Zeit von 8 bis 17 Uhr, Kinder über sechs Jahre bis zu drei Stunden täglich und in der Zeit von 8 bis 22 Uhr beschäftigt werden.

- Jugendmedienschutz: Klassisches Ziel des Jugendmedienschutzes ist es, dass Minderjährige nicht mit belastenden Medieninhalten in Kontakt kommen. Solche medieninduzierten Entwicklungsrisiken ergeben sich für unterschiedliche Altersstufen aus unterschiedlich drastischen, belastenden oder desorientierenden Darstellungen. Sowohl das Jugendschutzgesetz (JuSchG) im Bereich der Trägermedien als auch der Jugendmedienschutz-Staatsvertrag für Rundfunk und Telemedien (JMStV) sehen Altersbewertungen und entsprechende Alterskennzeichen vor und knüpfen altersabhängige Abgabe- bzw. Zugangsbeschränkungen an diese. Die gesetzlich vorgegebenen Altersgrenzen sind 6, 12, 16 und 18 Jahre. Mit Blick auf die fortschreitende Entwicklung werden Kindern und Jugendlichen bei der Alterseinstufung mit steigendem Alter zunehmende Kompetenzen beim Umgang mit und der Verarbeitung von auch fordernden jugendschutzrelevanten Darstellungen zugemutet.

- Zivilrechtliche Geschäftsfähigkeit: Ein weiteres Beispiel für gesetzgeberische Altersabstufungen bei Kindern und Jugendlichen ist die Regelung der Geschäftsfähigkeit Minderjähriger im Bürgerlichen Gesetzbuch (BGB). Schutzgedanke ist hier, dass Rechtsgeschäfte nachteilige 
Folgen für die Rechtsposition und das Eigentum von Minderjährigen haben können. Zentral unterstellt der Gesetzgeber hier Defizite in der Möglichkeit der eigenverantwortlichen Willensbildung (Spickhoff 2018); es geht um einen „Schutz vor sich selbst“. Nach den $\$ \mathbb{S} 104 \mathrm{ff}$. BGB sind Kinder unter sieben Jahren geschäftsunfähig, d.h. sie können keine wirksamen Willenserklärungen abgeben und keine Rechtsgeschäfte abschließen. Kinder zwischen sieben und 17 sind beschränkt geschäftsfähig, d.h. sie können Willenserklärungen abgeben und (ausschließlich vorteilhafte) Rechtsgeschäfte vornehmen. Bis zu der elterlichen Genehmigung sind geschlossene Verträge schwebend unwirksam. Nach dem sog. „Taschengeldparagraphen“ ( $\$ 107$ BGB) können Minderjährige zwischen sieben und 17 Jahren ausnahmsweise auch ohne elterliche Zustimmung verbindliche Verträge schließen, wenn sie zur Bewirkung der Leistung Mittel nutzen, die dem Minderjährigen zu diesem Zweck oder zur freien Verfügung überlassen wurden.

- Datenschutzrechtliche Einwilligung: Das EU-Datenschutzrecht geht davon aus, dass Kinder bei der Verarbeitung ihrer Daten eines besonderen Schutzes bedürfen, vor allem mit Blick auf die spezifischen Gefahren der Datenverarbeitung für den Datenschutz, aber auch für die Ausübung von anderen Grundrechten und Freiheiten (sog. Vorfeldschutz). Art. 8 DSGVO sieht vor, dass eine Einwilligung gegenüber OnlineDiensten in die Verarbeitung der eigenen personenbezogenen Daten durch Kinder und Jugendliche unter 16 Jahren stets durch die Eltern erfolgen muss. Außerhalb dieser im Vergleich starren Regelung der Einwilligung gegenüber Diensten der Informationsgesellschaft gilt weiterhin der Grundsatz, dass die individuelle Einsichtsfähigkeit Voraussetzung einer wirksamen datenschutzrechtlichen Einwilligung ist. Auch mit Blick auf die angenommene Einsichtsfähigkeit in die teils komplexen Datenverarbeitungsvorgänge geht die Rechtswissenschaft davon aus, dass diese mit dem fortschreitendem Alter Heranwachsender zunimmt.

Die ausgewählten Beispiele zeigen, dass das Recht vielfach abgestufte Schutzräume und -vorkehrungen für Kinder und Jugendliche vorsieht bzw. einzieht. Die gesetzgeberischen Annahmen entsprechen dabei den oben beschriebenen Erkenntnissen der Entwicklungspsychologie: Mit zunehmendem Alter traut das Recht Minderjährigen mehr Einsichtsfähigkeit und Verantwortung zu. (s. die vereinfachte Abb. 1). Der besondere Schutz Minderjähriger im Recht bezieht sich dabei auf zeitliche, physische, psychische, aber auch soziale Schutzräume, in denen Kinder und Jugendliche 
aufwachsen und ihre Persönlichkeit möglichst unbeeinträchtigt entwickeln (sollen).

Abb. 1: Biologische Entwicklung und Abstufungen rechtlicher Schutzräume (vereinfacht)

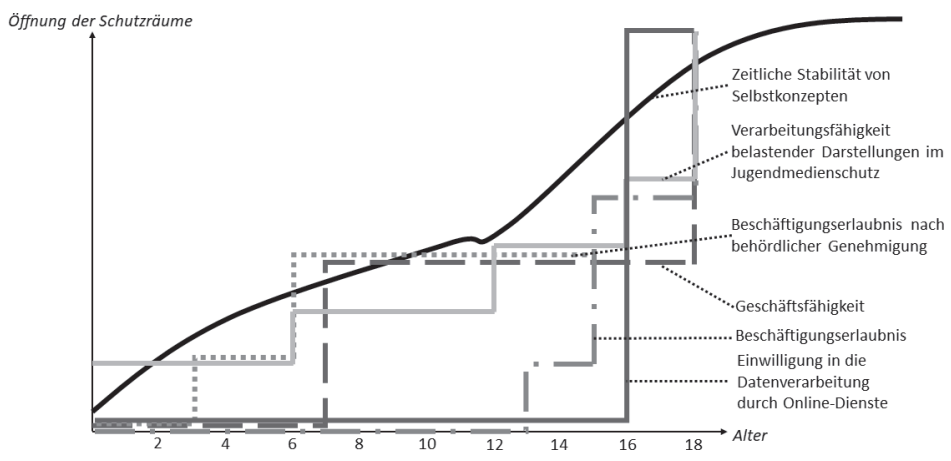

4. Privatheits- und autonomiebezogene Zielaspekte bei der Gewährleistung unbeeinträchtigten Aufwachsens

Die Anschlussfrage mit Blick auf das Schutzziel einer möglichst unbeeinträchtigten Persönlichkeitsentwicklung ist, inwieweit dieses Ziel auch autonomie- und privatheitsbezogene Aspekte beinhaltet. Das Recht auf unbeeinträchtigte Persönlichkeitsentwicklung betrifft angesichts ihrer besonderen Entwicklungsphase und der daraus folgenden besonderen Ausgestaltung rechtlicher Schutzräume vor allem heranwachsende Kinder und Jugendliche. Nicht umsonst erscheint die Freiheit der Persönlichkeitsentwicklung als Kern des verfassungsrechtlichen Kinder- und Jugendschutzes. Deswegen lohnt ein zweiter Blick auf dessen Schutzziele:

Kernelemente des Ziels einer freien Persönlichkeitsentwicklung und -entfaltung von Minderjährigen sind nach Ansicht des BVerfG die Gemeinschaftsfähigkeit und die Eigenverantwortlichkeit. ${ }^{1}$ Hier konkretisiert sich die Entwicklungsfreiheit als ein nicht zum Selbstzweck gewährtes Recht, sondern als Aufrechterhaltung eines normativen Nexus an Entwicklungsmöglichkeiten, dessen Zielerreichung der Staat sich wünschen, aber selbst nicht positiv garantieren kann. Der Schutzauftrag soll dann wenigstens die auf diese Erreichung negativ wirkenden Einflüsse fernhalten - die

1 BVerfGE 79, 51 (63 f.); 83, 130 (139). 
Gewährleistung von Entwicklungsfreiheit erfolgt um die Möglichkeit, mündige Bürger"innen zu werden.

Das grundgesetzlich erwünschte Entwicklungsziel der Eigenverantwortlichkeit verweist auf ein „Verständnis der Persönlichkeitsentwicklung als autonomer Prozess, als Möglichkeit, das eigene Selbstbild nach eigenem Entwurf zu gestalten, ohne dass externe Einflüsse in diese Entwicklung eingreifen" (Dreyer 2018a: 202). In diesem Verständnis von Eigenverantwortlichkeit als Selbstbestimmtheit scheint das oben angesprochene Recht auf sein "Selbst“ und die damit verbundene Autonomie deutlich auf. Der Gewährleistungsauftrag für Autonomie im Sinne einer Selbstbestimmtheit zielt ab auf den Schutz gegen Fremdbestimmtheit, etwa in Form von willentlicher Beeinflussung bis hin zu Fremdsteuerung. Der staatliche Jugendschutzauftrag umfasst so neben klassischen Entwicklungsrisiken, die Traumata, Ängste oder psychische Störungen und Zwänge auslösen können und sich als Einschränkung oder Hemmung eigenverantwortlicher Entscheidungen manifestieren, auch „weichere“ Beeinträchtigungen der Autonomie: Dort, wo autonome Entscheidungen oder autonomes Handeln durch von außen aufoktroyierte Werte, Sichtweisen oder Rollenverständnisse und ohne Möglichkeit des Erlernens kritischer Reflektion, Hinterfragung und Offenheit für andere, alternative Sicht- und Entscheidungsweisen eingeschränkt werden, muss der Schutzauftrag ebenfalls aktiviert sein (Dreyer 2018a: 157).

\subsection{Verschränkung von Autonomie und Privatheit}

Für die Nutzung digitaler Medien durch Kinder und Jugendliche muss vom Autonomieschutz ausgehend auch dessen enge Beziehung zu Privatheit und Privatheitskonzepten diskutiert werden. Autonomie, verstanden als das Recht und die Möglichkeit, über sein Selbst zu bestimmen, benötigt Privatheit: Es bedarf individueller Räume, in denen sich die eigene Persönlichkeit entwickeln kann (Rössler 2018: 93). Privatheit als Privatsphäre umschreibt den Raum, in dem sich autonome Selbstentwicklung vollziehen kann und vollzieht - sie ist „das herausgehobene Refugium der Selbstverwirklichung" (Weiß/Groebel 2013: 19). Wie oben gezeigt entwickelt sich Identität aber - und vor allem - auch durch soziale Interaktion. Diese Interaktion kann auch digital vermittelt sein. Es reicht für das Privatheitsverständnis nicht, nur auf eine engere persönliche physische Sphäre abzustellen, sondern auch weiterreichende soziale Räume unter den Privatheitsbegriff zu subsumieren. Rössler und Trepte haben den sozialen Aspekt von (relationaler) Privatheit herausgearbeitet (Rössler 2001: 
234-251, Rössler 2018, Trepte 2016). Weitere neue Privatheitskonzepte weisen zudem auf das Verhältnis von Privatheit, Autonomie und Demokratie als supraindividuelle, gesamtgesellschaftliche Wirkungsdimensionen hin (Becker 2017a, Becker/Seubert 2016, Gusy 2015). Koops et al. haben 2017 die vielschichten Privatheitsdimensionen entlang ihrer jeweils relevanten Sphäre und ihrer freiheitsbezogenen Schutzrichtungen in einer relativ umfassenden Matrix aufgespannt (s. Abb. 2), die nachvollziehbar aufzeigt, wieso Privatheit und Autonomie auch bei digitaler Mediennutzung umfassend berührt sind.

Abb. 2: Typologisierung von Privatheitskonzepten („A typology of privacy“)

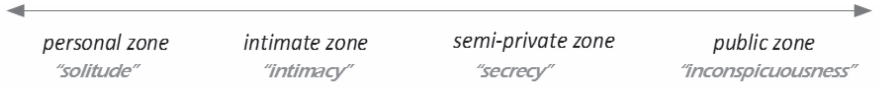

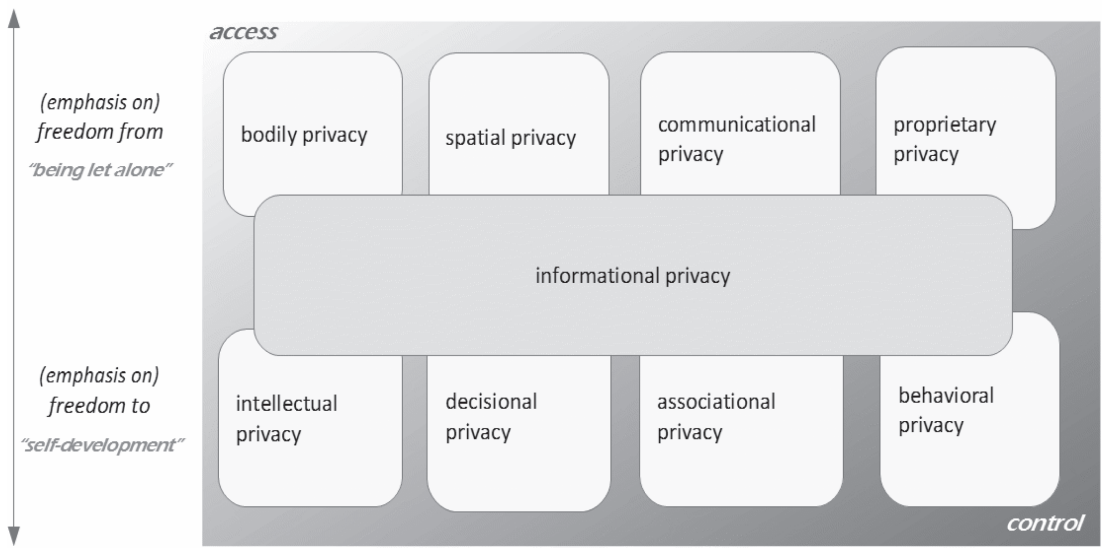

Quelle: Koops/Newell/Timan/Škorvánek/Chokrevski/Galič 2017: 566.

In der Zusammenschau des Spektrums unterschiedlicher Persönlichkeitssphären (höchstpersönlich/intim, privat, sozial, öffentlich) und den unterschiedlichen freiheitsbezogenen Schutzrichtungen (Freiheit von etwas, und Freiheit zu etwas - hier in erster Linie die der Persönlichkeitsentwicklung) liegt der Aspekt der informationellen Privatheit quer über alle Sphären und berührt sowohl die negative als auch die positive Ausprägung eines Rechts auf Privatheit. Über medial vermittelte Kommunikation können alle Formen der intimen, persönlichen, sozialen und öffentlichen Formen der Persönlichkeitsentwicklung und -entfaltung berührt sein; informationelle Privatheit erscheint daher als fundamental wichtig „in allen sozialen Bezügen, in denen Subjekte leben“ (Rössler 2003: 33). 


\subsection{Reichweite informationeller Privatheit bei der digitalen Mediennutzung Heranwachsender}

Anhand einiger exemplarischer Bereiche sollen die vielfältigen Berührungspunkte informationeller Privatheit bei der Nutzung digitaler Medien und deren Autonomiegesichtspunkte aufgefächert werden. Ausgangspunkt der folgenden Überlegungen ist der Umstand der Rückkanalfähigkeit digitaler Kommunikation, und damit die Möglichkeit der Beobachtbarkeit. Durch die Nutzung digitaler Kommunikationsmedien fallen nicht nur die Kommunikationsinhalte als Daten an, sondern auch die technischen Verkehrs- oder Nutzungsdaten in Form von Metadaten: Wer mit wem kommuniziert, von wo aus, worüber und wie lange; wer wann welche Angebote und Dienste nutzt; die Praxis der Beobachtung, Sammlung und Auswertung (auch) dieser Daten kommunikativer Kontakte ist weit verbreitet und Grundlage datenbasierter Monetarisierungsstrategien und Geschäftsmodelle. Durch die Auswertung von Inhalts- und Nutzungsdaten können Anbieter datenbasierte Gegenbilder in Form von Persönlichkeitsprofilen oder Segmenten generieren, die als Input in algorithmischen Selektionsverfahren wiederum Einfluss auf den Output gegenüber diesen Personen haben. Auf diese Weise entstehen Feedback-Loops auf Basis der mathematischen Normalisierung der Persönlichkeitsfacetten, der "Schubladisierung“ von Identitätsaspekten auch bei Heranwachsenden. Die digitalen Abbilder von Einzelnen sind dabei relativ statisch und können dynamische Entwicklungen und Veränderungen sowie Kontextspezifika nur schlecht abbilden. Dadurch entsteht das Risiko, dass die Profilierung von Heranwachsenden eher den „Schnappschuss" eines Stadiums der Persönlichkeitsentwicklung zu einem bestimmten Zeitpunkt und in einem bestimmten Kontext darstellen, der zügig durch neue oder erweiterte Selbstkonzepte oder Kontextveränderungen abgelöst wird und damit nicht mehr valide ist. Durch die Zementierung digitaler Abbildung aber entstehen Formen digitaler Personae - „Datenschatten“ -, die nur zum Teil selbst kreiert sind, sondern durch externe Beobachtung ermittelt, berechnet und profiliert wurden (Roosendaal 2010). Die personalisierten Dienstleistungen entsprechen so nicht der tatsächlichen jeweiligen Persönlichkeit, sondern produzieren Ergebnisse auf Grundlage eines früheren, unscharfen Abbilds der Persönlichkeitsfacetten und Interessen der jeweils berechneten Person; sie sind zeitlich und kontextuell desintegriert. Durch Output auf Grundlage invalider Inputs aber werden ggf. Entwicklungsprozesse angestoßen, die sich ohne die Beobachtungspraktiken nicht realisiert hätten (zu den epistemischen Verschränkungen von Datenanalyse und Subjektentscheidung vgl. Albers 2017: 25f.). Der Autonomiebezug wird bereits hier deutlich. 
Auch die Frage der Kenntnis der Beobachtbarkeit und Beobachtung hilft bei Kindern und Jugendlichen nicht als moderierender Effekt: Haben minderjährige Nutzer*innen Kenntnis von der Beobachtung und Profilierung, so könnte dies bereits zu einem angepassten, jeweils vermeintlich sozialadäquaten Verhalten führen. Eine wirklich autonome Persönlichkeitsentwicklung wäre so nicht gewährleistet. Bei Nicht-Kenntnis von Profiling-Praktiken dagegen würden sich Minderjährige entsprechend unbeobachtet verhalten und damit ggf. besonders intime bzw. private Verhaltensmuster offenbaren. Diese aber wären Inbegriff der experimentellen Entwicklungsphase von Heranwachsenden, die nur deswegen an den Tag gelegt werden, weil man sich unbeobachtet fühlt. Beide Alternativen weisen insoweit deutliche Autonomiebezüge auf.

Mit der umfassenden Beobachtung und algorithmischen Informationsselektion erfolgt zudem eine autonomierelevante Aufmerksamkeitssteuerung (Dreyer/Heldt 2021). Durch predictive analytics werden die Wahrscheinlichkeiten relevanter - oder eher: aufmerksamkeits- und verweildauermaximierender - Informationen berechnet und entsprechende Informationen priorisiert. Dadurch entstehen niedrigschwellige, individualisierte Informationsangebote und „Feeds“, die Einfluss auf die Entscheidungsfreiheit nehmen. Die gleichen Algorithmen steuern Teile des sozialen Austauschs und der Selbstdarstellung auf Plattformen und darüber hinaus: Sie entscheiden für den Einzelnen, welche Informationen, Updates und Likes von welchen Freunden und Bekannten zu sehen sind - und welche nicht. Diese Selektion erfolgt auch hinsichtlich der eigenen Informationen, und welche der eigenen Freund"innen und Bekannten diese Informationen von mir bzw. über mich sieht. Damit weisen diese Praktiken auch Berührungspunkte zum eigenen Beziehungs- und Identitätsmanagement (Schmidt 2006, Schmidt 2017) auf: Nicht mehr der Einzelne entscheidet, wer was wann über ihn weiß, sondern die Selektions- und Rankingalgorithmen von Plattformen. Aufmerksamkeitssteuerung durch Algorithmen ist auch Steuerung von sozialen Beziehungen, Kontexten und wahrgenommener Identität (Thimm/Bächle 2019: 80, Einspänner-Pflock 2017: 101). Beides - die aufmerksamkeitsbezogene Steuerung der Inhalte anderer und die Steuerung der Rezeption der eigenen Inhalte bei anderen - hat hohe Relevanz für die Persönlichkeitsentwicklung und -entfaltung (Becker 2017b).

Deutlich wird bei den hier aufgezeigten autonomierelevanten Praktiken der Beobachtung und Aufmerksamkeitssteuerung im Digitalen, dass unterschiedliche Arten und Herkunftssphären von Daten dabei eine Rolle spielen (s. Abb. 3): Teile der Beobachtung und Auswertung fußen auf bewusst selbst preisgegebenen Informationen von Kindern und Jugendlichen, wei- 
tere erfolgen auf Grundlage unbewusst preisgegebener bzw. beiläufig erzeugter Informationen im Rahmen der Mediennutzung. Daneben können auch Dritte (Eltern, Freunde, Bekannte, Unbekannte) die Ursache für die Preisgabe von Daten gebildet haben - etwa durch absichtlich offengelegte Informationen oder durch unbewusst entstandene Daten im Rahmen der Kontaktaufnahme oder durch die Auswertung von Kommunikationsinhalten.

\section{Abb. 3: Kommunikations- und Beobachtungsebene}

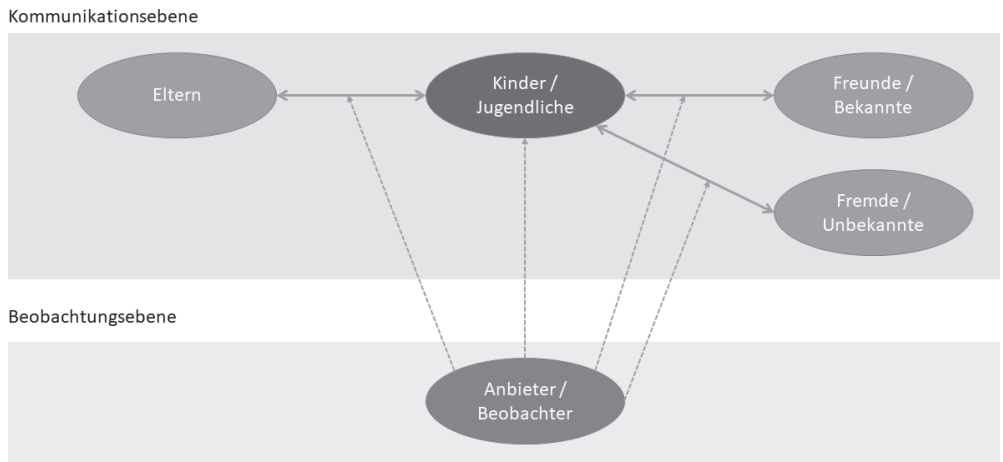

\section{Digitale Mediennutzung von Kindern als Experimentierraum der}

Persönlichkeitsentwicklung: Regulierungsnotwendigkeit und

Steuerungsherausforderungen

Der kurze Überblick hat gezeigt, dass bei der digitalen Mediennutzung von Kindern und Jugendlichen eine Reihe von Beobachtungs- und Auswertungspraktiken stattfindet, die Berührungspunkte zu Autonomie aufweisen. Dabei scheinen unterschiedliche Schutzdimensionen auf: Erstens nutzen Heranwachsende Kommunikationsmedien als digitale Experimentierräume der Persönlichkeitsentwicklung. Die Beobachtung dieses Aufwachsens in Online-Umgebungen erscheint grundsätzlich als Eingriff in die besonders schützenswerte Privatsphäre von Kindern und Jugendlichen. Zweitens sind die dabei entstehenden digitalen Gegenbilder und Persönlichkeitsprofile angesichts der fluiden Selbstkonzepte bei Heranwachsenden schnell nicht mehr valide. Durch die Speicherung nicht mehr aktueller Persönlichkeitsprofile und der Selektion von darauf basierenden Einzelinhalten wird Einfluss genommen auf das Informations-, Identitäts- und Beziehungsmanagement der minderjährigen Nutzer*innen und so eben- 
falls das Recht auf unbeeinträchtigte Persönlichkeitsentwicklung berührt. Und drittens weisen prädiktive Verfahren Potenziale für autonomierelevante self-fulfilling prophecies auf, wenn sie sich zwischen profilbasierten Anzeige- und empfängerseitigen Auswahlentscheidungen zu Selbstverstärkungseffekten hochschaukeln (Dreyer 2018c).

Diese drei Aspekte verweisen auf den staatlichen Schutzauftrag im Jugendschutz, dennoch stellt sich die Frage, ob eine gesetzliche Intervention in der Folge notwendig wird? Das wäre zu bejahen, wenn es einen Schutzbedarf von Kindern und Jugendlichen gibt. Von einem solchen Bedarf ist wie gezeigt dann auszugehen, wenn die Heranwachsenden keine Möglichkeit haben, den autonomierelevanten Praktiken zu entgehen, oder gar keine Kenntnis von diesen Verfahren haben. Ihnen fehlte dann dasjenige Wissen, das für eine kritische Reflexion und ein entsprechend angepasstes, autonomes Handeln in der Nutzungspraxis erforderlich wäre.

\subsection{Kenntnis der Datenverarbeitungspraktiken und datenreflektiertes Handeln}

Für die Bestimmung eines möglichen Auslösens des gesetzgeberischen Schutzauftrags im Bereich eines spezifischen Kinderdatenschutzes ist es notwendig, den Blick über theoretische Ansätze hinaus (Peter/Valkenburg 2011) auch auf empirische Untersuchungen zu werfen, die Evidenzen hinsichtlich der Kenntnisse von Datenverarbeitungspraktiken von Kindern und Jugendlichen zu Tage gefördert haben und Hinweise auf ein entsprechend geringes oder hohes datenreflektiertes Handeln liefern. Die einschlägigen Erhebungen (Schenk et al. 2012, Trepte/Masur 2015, DIVSI/ SINUS 2014, BITKOM 2014, Livingstone et al. 2018) weisen im Kern und entsprechend der oben beschriebenen auch kognitiven Entwicklungen in Kindheit und Jugend eine deutliche Altersabhängigkeit der datenbezogenen Kenntnisse und des Handelns auf. ${ }^{2}$ Der Einfachheit halber differenziert der Beitrag hier die Altersgruppen der 0-6-Jährigen, der 7-13-Jährigen und der 14-18-Jährigen:

- Die Untersuchungen zum Privatheitsverständnis von kleineren Kindern (0-6 Jahre) weisen darauf hin, dass diese Altersgruppe in der Regel

2 Die hier gemachten Aussagen beruhen zusätzlich auf den Vorabergebnissen qualitativer Befragungen von Kindern und Jugendlichen zu Privatsphäre und Datenschutz im Herbst 2019 im Rahmen des Projekts „Datafied Childhood“ von Stephan Dreyer, Claudia Lampert und Kira Thiel; die Ergebnisse sind noch nicht veröffentlicht. 
nur ein binäres Konzept von Privatheit entwickelt hat. Unterschieden wird von Kindern in dieser Altersgruppe nur zwischen Alleinsein versus Zusammensein (Livingstone et al. 2018, Birkner 2005).

- Die Befragungen von älteren Kindern (7-13 Jahre) zeigen, dass in diesem Alterssegment ein Verständnis von Privatheit grundsätzlich vorhanden ist (Schenk/Niemann/Reinmann/Roßnagel 2012, Livingstone et al. 2018). Hier entwickeln sich Konzepte unterschiedlich privater Räume und Formen verschieden privater und offener Sphären, die stark kontextabhängig sind. Dieses Grundverständnis wird in Ansätzen auf digitale Medienumgebungen übertragen, erfolgt dort aber fokussiert auf Formen relationaler Privatheit, d.h. die Einordnung des Privatheitsgrades von Informationen und Interaktionen erfolgt praktisch ausschließlich auf Grundlage des bekannten oder vermeintlichen Gegenübers, z.B. Verwandte, Freund"innen, Bekannte, Unbekannte (Einspänner-Pflock 2017, Litt 2012). In dieser Altersgruppe erlangen die meisten Kinder erste Kenntnisse von Überwachbarkeit im Netz generell, haben teilweise etwas von Verfolgbarkeit oder Beobachtbarkeit durch „Fremde“ gehört, ordnen dies aber noch nicht regelmäßig einzelnen Akteuren zu.

- Bei Jugendlichen ab 14 Jahren wird deutlich, dass die Heranwachsenden bereits eine gute Kenntnis der grundsätzlichen Problematik von Überwachbarkeit und Auswertbarkeit digitaler Mediennutzung haben (DIVSI/SINUS 2014). Sie weisen regelmäßig eine starke Erwartungshaltung in Richtung einer kontextuellen Privatheit auf, d.h. sie bewerten Privatheit vor allem anhand des Geheimnisinteresses einer bestimmten Information oder Konversation gegenüber Personen und Akteur"innen, denen das Wissen darüber nicht zusteht bzw. zustehen sollte (Nissenbaum 2010, Livingstone et al. 2018:19). Dabei nutzen sie meist einfache Risikoheuristiken, die bei einleuchtenden Affordanzen und kommunikativen Netzwerkeffekten aber schnell in den Hintergrund treten können: „Eigentlich gefällt mir nicht, dass jemand beobachten kann, mit wem ich worüber spreche, aber auf bestimmten Plattformen erreiche ich meine besten FreundInnen am einfachsten." Auch die Risikozuschreibung an einzelne Akteure zeigt die Oberflächlichkeit des Wissens um Datenverarbeitungspraktiken: Aus Sicht der Jugendlichen ist es in der Regel der App-Anbieter, der Webseitenanbieter, der SpielePublisher, der die Nutzer beobachtet. Die Kenntnis angebotsübergreifender Möglichkeiten der systematischen Beobachtung, Auswertung und Profilierung (WhatsApp/Instagram/Facebook; FitBit/Youtube/ Google) ist nur einzeln festzustellen. Das Wissen um komplexere angebots- und geräteübergreifende Tracking-Techniken, die sich einer Viel- 
zahl ineinandergreifender Dienste und Anbieter (insbesondere sog. ad services) bedient, fehlt in diesem Alterssegment praktisch völlig.

Der Abgleich von Schutzzielen und den Hinweisen auf die aktuelle Kenntnis schutzzielrelevanter Praktiken und einem entsprechenden Handeln weist durchgängig signifikante Diskrepanzen auf. Insbesondere durch die systematische Beobachtung und Sammlung kommunikationsbezogener (Meta-)Daten bei der Mediennutzung Minderjähriger entstehen Gefährdungen für die Gewährleistung einer freien und unbeeinträchtigten Persönlichkeitsentwicklung. Dieses Risikopotenzial entsteht zum einen durch ein wachsendes Bewusstsein bezüglich des Beobachtet-Seins bei Heranwachsenden, was nicht nur theoretisch zu einem vermeintlich sozial angepassten - und damit unfreiem - Online-Verhalten führen kann. Zum anderen können durch die systematische Beobachtung und eine darauf basierende Persönlichkeitsprofilierung Situationen entstehen, in denen Selektionen, Ausspielungen, Priorisierungen, aber auch De-Priorisierungen zu einer algorithmisierten „De-Autonomisierung“ der Persönlichkeitsentwicklung führen können. Die Persönlichkeitsfacetten Minderjähriger entwickeln sich (schlimmstenfalls) in jene Richtungen, die von profilbildenden Algorithmen berechnet und in Selbstverstärkungskaskaden zementiert werden. Gegen derartige Effekte haben Kinder und Jugendliche wenig Handhabe, wenn sie kaum etwas über die Umstände ihrer Herkunft und Entstehung wissen und keine entsprechenden Reflexionsebenen oder Abwehrstrategien entwickeln können. Neben den autonomiebezogenen Beeinträchtigungen scheinen auch hier letzten Endes demokratiebezogene Mündigkeitsproblematiken auf (vgl. Berson/Berson 2006).

\subsection{Möglichkeiten und Grenzen rechtlicher Steuerung}

Der Auftrag an die Gesetzgebung, eine freie Persönlichkeitsentwicklung auch im Digitalen zu gewährleisten, erscheint angesichts der empirischen Evidenzen gerechtfertigt. Die anschließende Frage, die sich stellt, ist dann, ob und in welcher Form gesetzliche Vorgaben hier helfen können - und mit welchen Steuerungsinstrumenten dies umgesetzt werden könnte. Die rechtliche Bearbeitung von Privatheitsrisiken bietet sich durch Interventionen insbesondere mit Blick auf die oben gezeigten vier Akteurskonstellationen an (Abb. 3): Erstens im Verhältnis von Kind und datenverarbeitenden Anbietern eines Dienstes, zweitens im Verhältnis von Kind und Erziehungsberechtigten, drittens im Verhältnis von Kind und befreundeten oder bekannten Kommunikationspartner*innen und viertens im Verhält- 
nis von Kind und fremden oder unbekannten Kommunikationspartner*innen. Doch bei jeder der vier Konstellationen sieht sich rechtliche Steuerung (unterschiedlichen) komplexen Herausforderungen gegenüber:

- Auf den ersten Blick vielversprechend erscheint es, wenn gesetzliche Regelungen an die beobachtenden bzw. datenverarbeitenden Anbieter eines Onlinedienstes, den Kinder und Jugendliche nutzen, anknüpfen. Hier könnten Beschränkungen (z.B. Verarbeitungsverbote), besondere Vorgaben (z.B. kindgerechte Voreinstellungen) oder Transparenzpflichten genau gegenüber jenen Akteur*innen gemacht werden, die den ersten Zugriff auf die genutzte Technologie und Infrastruktur haben. Alternativ könnte der Gesetzgeber das Mindestalter für die Nutzung entsprechender Dienste vorgeben. Dieser Ansatz aber weist Problempotenziale auf, da der Anbieter dafür genau wissen müsste, welche der Nutzenden eine minderjährige Person ist. Folgen müssten also Maßnahmen, die Heranwachsende online als solche erkennbar machten oder im Rahmen von Altersüberprüfungsverfahren zwingend neue Datensätze produzieren würden, z.B. Datenerhebung zu Zwecken des Datenschutzes. Auch die Online-Erkennbarkeit des Umstandes, dass es sich bei Nutzer*innen um eine minderjährige Person handelt - ggf. sogar mit Informationen zum konkreten Alter oder einer bestimmten Altersgruppe - hat aus Sicht des Jugendschutzes mehr Nach- als Vorteile (Dreyer 2018b). Zum Datenschutzdilemma hinzu träte also ein Erkennbarkeitsdilemma: Der Steuerungsansatz für einen besseren Datenschutz könnte im Extrem sogar zu einer Verschlechterung des Kinderschutzes führen.

- Auch ein gesetzliches Intervenieren in das Verhältnis von Kindern und Eltern, etwa durch die Konkretisierung elterlicher Schutzpflichten oder gar Untersagungsverbote bezüglich der kindlichen Nutzung bestimmter Dienste könnte zu einem Dilemma führen: Zum einen stehen $\mathrm{Fa}$ milienbeziehungen unter besonderem (Privatheits-)Schutz. Intrafamiliale Beziehungen bilden aus Sicht des Rechts zunächst eine Sphäre der rechtsaversen Selbstgestaltung und -verständigung. Es gilt das Primat des Erziehungsrechts der Eltern, vor allem gegenüber Eingriffen und Bevormundung durch den Staat. Untersuchungen zeigen allerdings eine hohe Zahl von Eltern, die Handlungserwartungen ausdrücklich an den Staat und die Anbieter*innen formulieren (Brüggen et al. 2017). Soweit man dadurch bereits in das Gebiet des Wächteramts des Staates als Kompensation elterlicher Motivations- oder Möglichkeitsausfälle bei der Übernahme ihrer Erziehungsverantwortung gelangen sollte (Dreyer 2019), müsste man in Kauf nehmen, dass der"die Gesetzge- 
ber*in hier den Bereich unmittelbarer staatlicher Steuerung von Familienbeziehungen und Erziehungspraktiken regulieren würde (Dreyer 2018a: 191).

- Rechtliche Anknüpfungspunkte an Kommunikationen im Verhältnis von Minderjährigen zu ihren Freund*innen und Bekannten stoßen mit Blick auf das Fernmeldegeheimnis an verfassungsrechtliche Grenzen: Digital vermittelte Individualkommunikation ist ein Bereich, der besonders vor der Einsichtnahme durch den Staat und Dritte geschützt ist. Das Dilemma staatlicher Regelungen besteht hier darin, dass die Gesetzgebung etwas regeln würde, was der Staat nicht sehen soll.

- Ähnlich verhält es sich mit Regulierungsansätzen, die im Verhältnis von Heranwachsenden und unbekannten Dritten Wirkungen entfalten sollen. Auch hier besteht das Problem der vor Einsichtnahmen geschützten Individualkommunikation. Es bliebe theoretisch die Möglichkeit, die Offenbarung von Daten Minderjähriger durch Dritte strafrechtlich zu sanktionieren (über den Bereich hinaus, der derzeit von

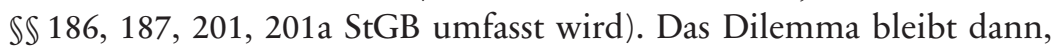
dass klassisches Strafrecht nicht als unterstützende Steuerungsressource im Vorfeld von Datenweitergabe wirkt, sondern erst als repressive Sanktionierung.

\section{Fazit}

Aufwachsen heißt, sich auszuprobieren - auch im digitalen Raum. Die bei der Beobachtung dieser Interaktionen anfallenden Daten zeigen domänenspezifische Facetten von sich im Fluss befindlichen Selbstkonzepten der Heranwachsenden. Sie manifestieren sich durch die Beobachtungs- und Auswertungspraktiken im Netz in datenbasierten Gegenbildern, die relativ statisch, dekontextualisiert und kontextübergreifend vorgehalten werden. Die Profile dienen algorithmischen Selektionsverfahren als Input und bestimmen auf dieser Grundlage den Output automatisierter Verfahren, mit Einfluss auf die wahrgenommenen Informationen über die Welt, über Freund*innen und Dritte, und die Wahrnehmbarkeit eigener Informationen auf der Seite von Dritten. Damit weist die Beobachtbarkeit der Mediennutzung in mehrfacher Hinsicht Autonomiebezüge auf: Allein das Bewusstsein oder die Sorge um die Beobachtbarkeit der Kommunikation kann zu Verhaltensanpassungen führen. Die gewollte Unkenntnis der Beobachtbarkeit ist mit Blick auf zu sichernde Autonomie keine Alternative. Unabhängig von einer solchen Kenntnis potenzieren, prädiktieren und normalisieren algorithmische Selektionsverfahren auf Grundlage von Be- 
obachtungsdaten die Persönlichkeitsentwicklung Heranwachsender. Sie weisen durch ihre Einflüsse auf das Informations-, Identitäts- und Beziehungsmanagement Heranwachsender ein hohes Beeinträchtigungspotenzial für die Persönlichkeitsentwicklung auf. Rechtliche Steuerungsmöglichkeiten zur Gewährleistung einer unbeeinträchtigten Persönlichkeitsentwicklung geraten hier indes gleich in mehrere Dilemmata; rechtliche Steuerung hat nur begrenzte Möglichkeiten. Wo es aber als Steuerungsressource genutzt werden kann, werden radikalere Vorgaben und konsequentere Durchsetzung dringend nötig.

Als spezifisch kinderbezogene Handlungsoptionen, durch die sich der Staat den aufgezeigten Regulierungsherausforderungen stellen könnte, erschienen vor allem ein Verbot nutzungsbasierten Trackings und Profilings, der Nicht-Einsatz prädiktiver Verfahren, altersangemessene Transparenzformen, kontextsensitive Informationen und „Tabula Rasa“-Möglichkeiten während und zum Ende der Kindheit.

\section{Literatur}

Albers, Marion (2017): Informationelle Selbstbestimmung als vielschichtiges Bündel von Rechtsbindungen und Rechtspositionen. In: Friedewald, Michael / Lamla, Jörn / Roßnagel, Alexander (Hg.): Informationelle Selbstbestimmung im digitalen Wandel. Wiesbaden: Springer, S. 11-35.

Andresen, Sabine (2018): Kindheit. In: Böllert, Katrin (Hg.): Kompendium Kinderund Jugendhilfe. Wiesbaden: Springer, S. 365-379.

Becker, Carlos (2017a): Privatheit und kommunikative Freiheit im Internet. In: Jacob, Daniel / Thiel, Thorsten (Hg.): Politische Theorie und Digitalisierung. BadenBaden: Nomos, S. 45-82.

Becker, Carlos (2017b). Kritische Theorie des Privaten. In: Friedewald, Michael / Lamla, Jörn / Roßnagel, Alexander (Hg.): Informationelle Selbstbestimmung im digitalen Wandel. Wiesbaden: Springer, S. 147-168.

Becker, Carlos / Seubert, Sandra (2016): Privatheit, kommunikative Freiheit und Demokratie. In: Datenschutz und Datensicherheit (DuD) 2, S. 73-78.

Berson, Ilene R. / Berson, Michael (2006): Children and Their Digital Dossiers: Lessons in Privacy Rights in the Digital Age. In: International Journal of Social Education 21 (1), S. 135-147.

Birkner, Enrico (2005): Auswirkungen der Raumstruktur eines Kindergartens auf das kindliche Verbalten und Erleben. Dresdner Arbeiten zur Architekturpsychologie. Online verfügbar unter: http://architekturpsychologie-dresden.de/ddarbeiten/bir kner_kindergarten.pdf (Abfrage am: 10.06.2020). 
BITKOM (Hg.) (2014): Jung und vernetzt. Kinder und Jugendliche in der digitalen Gesellschaft. Berlin. Online verfügbar unter: https://www.bitkom.org/noindex/Publ ikationen/2014/Studien/Jung-und-vernetzt-Kinder-und-Jugendliche-in-der-digita len-Gesellschaft/BITKOM-Studie-Jung-und-vernetzt-2014.pdf (Abfrage am: 10.06.2020).

Brüggen, Niels / Dreyer, Stephan / Drosselmeier, Marius / Gebel, Christa / Hasebrink, Uwe / Rechlitz, Marcel (2017): Jugendmedienschutzindex. Der Umgang mit onlinebezogenen Risiken. In: FSM - Freiwillige Selbstkontrolle MultimediaDiensteanbieter e.V. (Hg.): Ergebnisse der Befragung von Heranwachsenden und Eltern. Berlin.

Di Fabio, Udo (2019): Art. 2 I Rn. 2. In: Maunz, Theodor / Dürig, Günter (Hg.): Grundgesetz-Kommentar, 89. EL, Oktober 2019. München: Beck.

DIVSI/SINUS-Institut Heidelberg (2014): Kinder, Jugendliche und junge Erwachsene in der digitalen Welt. DIVSI U25-Studie. Hamburg: DIVSI. Online verfügbar unter: https:/www.divsi.de/wp-content/uploads/2014/02/DIVSI-U25-Studie.pdf (Abfrage am: 10.06.2020).

Dreyer, Stephan (2018a): Entscheidungen unter Ungewissheit im Jugendmedienschutz. Untersuchung der spielraumprägenden Faktoren gesetzgeberischer und behördlicher Entscheidungen mit Wissensdefiziten. Baden-Baden: Nomos.

Dreyer, Stephan (2018b): On the Internet, nobody knows you're a kid. Zur (Nicht-)Erkennbarkeit Minderjähriger in digitalen Medienumgebungen. In: Medien + Erziehung 62, S. 65-78.

Dreyer, Stephan (2018c): Predictive Analytics aus der Perspektive von Menschenwürde und Autonomie. In: Hoffmann-Riem, Wolfgang (Hg.): Big Data - Regulative Herausforderungen. Baden-Baden: Nomos, S. 135-143.

Dreyer, Stephan (2019): Rechte von Kindern und Jugendlichen, Elternprivileg und Wächteramt des Staates: Medienerziehung aus der Perspektive der Verfassung. In: Hajok, Daniel / Fleischer, Sandra (Hg.): Medienerziehung in der digitalen Welt. Grundlagen und Konzepte für Familie, Kita, Schule und Soziale Arbeit. Stuttgart: Kohlhammer, S. 86-102.

Dreyer, Stephan / Heldt, Amélie (im Druck): Algorithmische Selektion und Privatheit. Aufmerksamkeitssteuerung durch Social Media-Plattformen als Autonomieeingriff? In: Hennig, Martin et al. (Hg.): Verantwortung in digitalen Kulturen. Privatheit im Geflecht von Medien, Recht und Gesellschaft.

Dreyer, Stephan / Lampert, Claudia / Schulze, Anne (2014): Kinder und Onlinewerbung: Erscheinungsformen von Werbung im Internet, ihre Wabrnehmung durch Kinder und ihr regulatorischer Kontext. Leipzig: Vistas.

Einspänner-Pflock, Jessica (2017): Privatheit im Netz: Konstruktions- und Gestaltungsstrategien von Online-Privatheit bei Jugendlichen. Medien-Kultur-Kommunikation. Wiesbaden: Springer.

Greve, Werner / Thomsen, Tamara (2019): Entwicklungspsychologie: Eine Einführung in die Erklärung menschlicher Entwicklung. Wiesbaden: Springer.

Gusy, Christoph (2015): Privatheit und Demokratie. In: Kritische Vierjahreszeitschrift für Gesetzgebung und Rechtswissenschaft (KritV) 98 (4), S. 430-461. 
Koops, Bert-Jaap / Newell, Bryce Clayton / Tjerk, Timan / Chokrevski, Tomislav / Gali, Maša (2017): A Typology of Privacy. In: University of Pennsylvania Journal of International Law 38 (2), S. 483-575.

Litt, Eden (2012): Knock, Knock. Who's There? The Imagined Audience. In: Journal of Broadcasting \& Electronic Media 56(3), S. 330-345.

Livingstone, Sonia / Stoilova, Mariya / Nandagiri, Rishita (2018): Children's Data and Privacy Online: Growing up in a Digital Age: An Evidence Review. London: London School of Economics and Political Science. Online verfügbar unter: https:/www.lse.ac.uk/media-and-communications/assets/documents/research/pr ojects/childrens-privacy-online/Evidence-review-final.pdf (Abfrage am 3.10.2020).

Ochs, Carsten (2019): Teilhabebeschränkungen und Erfahrungsspielräume: eine negative Akteur-Netzwerk-Theorie der Privatheit. In: Behrendt, Hauke / Loh, Wulf / Matzner, Tobias / Misselhorn, Catrin (Hg.): Privatsphäre 4.0. Stuttgart: J.B. Metzler, S. 13-31.

Peter, Jochen / Valkenburg, Patti M. (2011): Adolescents' Online Privacy: Toward a Developmental Perspective. In: Trepte, Sabine / Reinecke, Leonard (Hg.): Privacy Online. Berlin / Heidelberg: Springer, S. 221-234.

Roosendaal, Arnold P. (2010): Digital personae and profiles as representations of individuals. In: Bezzi, Michele / Duquenoy, Penny / Fischer-Hübner, Simone / Hansen, Marit / Zhang, Ge (Hg.): Privacy and Identity Management for Life. Berlin, Heidelberg: Springer, S. 226-236.

Rössler, Beate (2001): Der Wert des Privaten. Frankfurt am Main: Suhrkamp.

Rössler Beate (2003): Anonymität und Privatheit. In: Bäumler, Herbert / von Mutius, Albert (Hg.): Anonymität im Internet. Wiesbaden: Vieweg+Teubner, S. 27-40.

Rössler, Beate (2018): Privatheit, Autonomie, Recht. In: Baer, Susanne / Sacksofsky, Ute (Hg.): Autonomie im Recht - Geschlechtertheoretisch vermessen. Baden-Baden: Nomos, S. 93-118.

Schenk, Michael / Niemann, Julia / Reinmann, Gabi / Roßnagel, Alexander (Hg.) (2012): Digitale Privatsphäre: Heranwachsende und Datenschutz auf sozialen Netzwerkplattformen. Berlin: Vistas.

Schmidt, Jan-Hinrik (2006): Social Software: Onlinegestütztes Informations-, Identitätsund Beziehungsmanagement. In: Forschungsjournal Soziale Bewegungen 19(2), S. 37-47.

Schmidt, Jan-Hinrik (2017): Das neue Netz: Merkmale, Praktiken und Folgen des Web 2.0. Konstanz: Halem.

Siegler, Robert S. / Eisenberg, Nancy / DeLoache, Judy S. / Saffran, Jenny (2016): Entwicklungspsychologie im Kindes- und Jugendalter. Berlin, Heidelberg: Springer.

Spickhoff, Andreas (2018): Vorbemerkung zu $\int \mathbb{S} 104 f f$. In: Säcker, Franz Jürgen et al.: Münchener Kommentar zum Bürgerlichen Gesetzbuch, 8. Auflage. Müchen: Beck. 
Thimm, Caja / Bächle, Thomas C. (2019): Autonomie der Technologie und autonome Systeme als ethische Herausforderung. In: Rath, Matthias / Krotz, Friedrich / Karmasin, Matthias (Hg.): Maschinenethik. Ethik in mediatisierten Welten. Wiesbaden: Springer VS, S. 73-87.

Trepte, Sabine / Masur, Philipp K. (2015): Privatheitskompetenz in Deutschland. Ergebnisse von zwei repräsentativen Studien. Bericht vom 18. November 2015. Stuttgart: Universität Hohenheim.

Trepte, Sabine (2016): Die Zukunft der informationellen Selbstbestimmung - Kontrolle oder Kommunikation? In: Stiftung Datenschutz (Hg.): Die Zukunft der informationellen Selbstbestimmung. Berlin: Erich Schmidt, S. 159-170.

Weiß, Ralph / Groebel, Jo (2013): Privatheit im öffentlichen Raum. Medienhandeln zwischen Individualisierung und Entgrenzung. Wiesbaden: Springer VS. 